\title{
An Approach to Pipe Image Interpretation Based Condition Assessment for Automatic Pipe Inspection
}

\author{
John Mashford, David Marlow, and Stewart Burn \\ Division of Land and Water, Commonwealth Scientific and Industrial Research Organisation, \\ P.O. Box 56, Highett, VIC 3190, Australia \\ Correspondence should be addressed to John Mashford, john.mashford@csiro.au
}

Received 5 March 2009; Accepted 11 September 2009

Recommended by Graham Sander

Condition assessment forms an important part of the asset management of buried pipelines. This is carried out through the use of inspection systems which usually consist of an image acquisition device attached to a mobile robotic platform. Complete or partial automation of image interpretation could increase the efficiency and objectivity of pipe inspection. A key component of an automatic pipe inspection system is the segmentation module. This paper describes an approach to automatic pipe inspection using pixel-based segmentation of colour images by support vector machine (SVM) coupled with morphological analysis of the principal component of the segmented image. The morphological analysis allows the principal component of the segmented image to be decomposed into the pipe flow lines region, the pipe joints, and adjoining defects. A simple approach to detecting pipe connections using fuzzy membership functions relating to defect size and location is also described.

Copyright (C) 2009 John Mashford et al. This is an open access article distributed under the Creative Commons Attribution License, which permits unrestricted use, distribution, and reproduction in any medium, provided the original work is properly cited.

\section{Introduction}

The safe transport, treatment, and disposal/reuse of sewage is integral to the healthy functioning of human society. The provision of these services relies on the continued functioning of numerous assets of many types, with design/service lives that range from very short to very long life (from a matter of years, to hundreds of years). In traditional sewerage systems, a network of sewer pipes is used to convey wastewater away from residential, commercial and industrial premises, to treatment plants where the waste is treated to the standard necessary for disposal to the environment or, increasingly, to allow water and solid constituents to be reclaimed and reused.

Sewer networks are generally large (in terms of the length of sewer mains), heterogeneous (in terms of asset characteristics and operating context), have a long life, and are subject to multiple and complex deterioration processes. Being buried, sewers are also hidden from view. All these factors mean that the management of sewerage systems presents a significant challenge to asset managers and maintenance professionals (see discussions on asset management in [1]). Asset management tools and processes are therefore needed to help target limited resources to where they can deliver most value in terms of maintaining asset function and service provision.

It is widely accepted that risk-based concepts provide an appropriate framework within which to address this management challenge [2]. In asset management, risk is normally taken to be the product of failure likelihood and consequence. However, since it is difficult to determine failure likelihood for wastewater assets [1], asset condition is often used as a surrogate measure (e.g., [3]). In this approach, acceptable asset condition states are defined that characterize the threshold above which the likelihood of failure is deemed unacceptable (e.g., [4]). Inspection of asset condition can then be used to determine whether the current state of an asset is acceptable or not; this process is generally termed "condition assessment" [3].

Given the potential expense of undertaking condition assessment across a sewer network, advances in both asset management processes and inspection technologies are being sought that will deliver lower cost inspection and/or allow greater value to be extracted from the inspection data provided. With these needs in mind, this paper presents some research into automation of inspection using digital 
images. A brief overview of sewer deterioration is first presented, along with a description of the CCTV inspection procedure widely used to identify that deterioration. The material is presented in such a way as to illustrate the role CCTV inspection plays in the management of sewer assets and thereby define the fundamental requirements for automating this process. The main part of the paper focuses on research into image recognition technology, which is being undertaken with the overarching aim of reducing the unit cost of CCTV inspection.

\section{Management of Sewer Deterioration}

The deterioration of gravity sewer pipes can be characterized as a time dependent process that moves through a number of distinct phases. In particular, there are at least three stages involved in progression towards structural failure of a pipe; these are (see also [5] and references given therein) the following:

(i) Damage: an initial, often minor, defect is imparted in some way.

(ii) Deterioration: progression of the damage then occurs through various deterioration mechanisms, such as the loss of soil support from around the pipe.

(iii) Structural failure: when it eventually occurs, structural failure is often triggered by some random event that may not be related to the deterioration process.

As sewer pipes are hidden from day to day view, deterioration can occur unnoticed and this can in turn lead to unexpected functional failures. The consequences of such failures range from minor impacts (interruption to customer service provision such as poor toilet flushing) to major ones (such as pollution incidents associated with overflows, internal flooding of properties, and even injury or loss of life associated with collapse of roads and other structures). As well as these consequences, the cost of responding to a failed sewer may be high due to the reactive nature of mobilising the necessary resources, equipment and material, and the additional expense this incurs in comparison to planned/programmed work. With these issues in mind, it can be economic to inspect assets to gain an understanding of the structural condition of a pipe (or group of pipes) and prevent failure through subsequent interventions (e.g., repair, renovation, or replacement of the asset). Such inspections are also undertaken for other asset management purposes, including regulatory reporting and strategic planning (e.g., [1]).

Close circuit television (CCTV) cameras are commonly used to inspect sewer pipes. The CCTV camera is inserted into the pipe to be inspected through a manhole or other access structure. The camera is then traversed along the pipe by manual or mechanical means. The image obtained is scrutinised by an operator and any defects along the pipeline noted, preferably using a standardised defect coding scheme (see [6] for more descriptive information and details on coding schemes). A recording of the inspection may also be assessed subsequently by an engineer, as deemed necessary.
To simplify reporting and summarise the results of the CCTV inspection, a common practice is to use the observed defects (directly based on operator judgement or through review of defect codes) to allocate an overall (summary) condition grade to the pipe section (a pipe section generally being defined in terms of the pipe length between two access structures). The grade can be allocated in terms of the worse defect observed, for example, a "peak score" and/or as an overall measure of defects along the pipe section, for example, a "mean score" (see $[6,7]$ for more details).

The allocated condition grade summarises the pipe's overall condition in terms of descriptive states ranging from the "as new" through to "derelict" condition. For example, a condition grade definition used in the WRc Sewer Rehabilitation Manual [7] is shown in Table 1. Such grading schemes can be conceptualised as a simplified representation of the sewer deterioration process; that is, the pipe is assumed to move from grade 1 to the worse condition grades as it deteriorates.

\section{Limitations of the CCTV Inspection Procedure}

It is broadly acknowledged that CCTV inspection is a subjective process that is influenced by operator error (e.g., see discussions in [1]). This subjectivity has been used by some researchers to justify the development of automated CCTV inspection systems (e.g., [8]). It is therefore interesting to consider whether this subjectivity truly provides justification for automation, or if there needs to be other drivers. With reference to Table 1, it can be seen that the detailed information gained from a CCTV inspection is ultimately summarised into simple categories that reflect the potential for failure, and thus the priority for maintenance work. Rather than obtaining an exact characterisation of the sewer's defects, a key requirement for routine CCTV inspections is thus to allocate assets to these grades appropriately. Some degree of subjectivity in the assessments is therefore acceptable, as long as the subjectivity does not impact on the decision to undertake maintenance interventions. Consider, for example, the practical interpretation of the grading scheme shown in Table 1. The definitions used imply that it is important for a CCTV operator or other assessor to identify defects that distinguish between assets in condition grades 1 to 3 (no work required) and those in condition grades 4 or 5 (work required immediately or in the foreseeable future).

With these considerations in mind, it is absolutely necessary that the interpretation of CCTV inspection data to be accurate enough to identify defects that indicate that an asset is condition grades 4 or 5 ; that is, that maintenance work is needed. Improvements in accuracy of the inspection process beyond this level of characterisation are, in the authors' opinion, unnecessary for general asset management purposes, and the subjectivity of the current inspection procedure does not therefore provide an appropriate level of justification for developing automated processes. Instead, it is our assertion that the process of using automation is only justified if it reduces the overall cost of inspection and 
TABLE 1: Example sewer grading scheme.

\begin{tabular}{ll}
\hline Structural grade & Interpretation \\
\hline 1 & Acceptable structural condition \\
2 & Minimal collapse risk in short term but potential for further deterioration \\
3 & Collapse unlikely in the near future but further deterioration likely \\
4 & Collapse likely in the foreseeable future \\
5 & Collapsed or collapse imminent \\
\hline
\end{tabular}

thereby allows more assets to be inspected. We envisage two levels of automation are possible that

(1) Fully automated. This represents the ideal; the inspection procedure would be fully automated and allow asset condition to be characterised accurately without the need for review by operators. Given our experience of the current state of the art and difficulties associated with the operating environment (especially the level of cleanliness of sewers and flow of sewage), we think that it is unlikely full automation will be achieved in the foreseeable future, if ever.

(2) Semiautomated. As a pragmatic step towards future automation, it may be possible to provide an automated screening function that determines assets that should be reviewed by operators. This would reduce the costs of reviewing CCTV inspections by operators.

With this later aim in mind, work has been undertaken into the development of image recognition technologies, which is described throughout the remainder of this paper.

\section{Image Recognition for Automatic Pipe Inspection}

Automatic pipe inspection systems consist of two parts. These are the image acquisition component and the image recognition, or interpretation, component. The image acquisition system is usually made up of a robotic device which moves through the pipe obtaining images. The images may be grey-scale, colour, range, or other sensor modality. The interpretation system processes images obtained by the image acquisition device identifying defects and features and, possibly, producing a report about the state of the pipe.

An early semiautomatic inspection system was the KARO system [9] which consisted of a multisensor inspection device and a two-pass interpretation system. During the largely automatic first pass a hierarchical fuzzy logic sensor fusion algorithm was used to identify candidate defects which were then investigated in detail by the operator during a second pass.

The PIRAT system [10] was made up of an inspection device providing range images (obtained by laser striping) together with an interpretation system using neural networks and other AI techniques [11]. The image acquisition device for the PIRAT system is shown in Figure 1. The system of Moselhi and Shehab-Eldeen [12] (in which the analysed

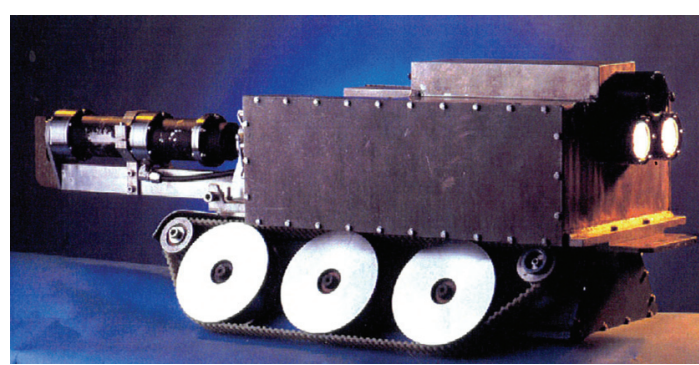

FIgURe 1: The PIRAT image acquisition device.

images were obtained from CCTV by a frame grabber) utilised a backpropagation neural network operating on feature vectors derived from defect regions. Sinha and Karray [13] describe a system for recognising pipe cracks using image analysis, and a neuro-fuzzy algorithm. The system of Duran et al. [14] operates on camera/laser images and uses a two-stage approach based on image processing and artificial neural networks. In the first stage a binary classifier identifies defective pipe sections and in the second stage the defects are classified into different types.

Our recent work has focussed on the development of an interpretation system rather than on the building of an inspection device. Data for such an interpretation system could be provided by a commercial inspection system such as that shown in Figure 2. Such an inspection system will move through a pipe and obtain a number of colour images. We also assume that there is the capability to combine together all of these images to form an unwrapped pipe image. An unwrapped pipe image is an image of the pipe represented in cylindrical polar coordinates. Figure 3 shows part of such an image. In the present paper we will assume that the interpretation system has access to such an unwrapped pipe image. If this were not the case then it would be necessary to either construct an unwrapped pipe image from the underlying images and then process that or else to process each of the individual underlying images and then to weave together all of the processing outputs to form an overall pipe condition report.

The colour images provide significantly different information from grey-scale images or range images. This can augment the effectiveness of some of the image processing tasks that are to be carried out on the images. However, in some other respects, the tasks are more difficult. With colour images, where the information available is that of 


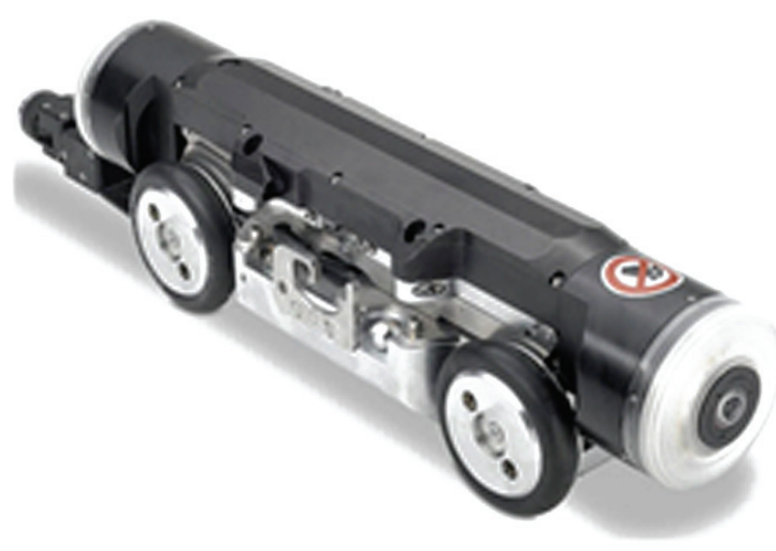

FIgURE 2: A commercial inspection system.

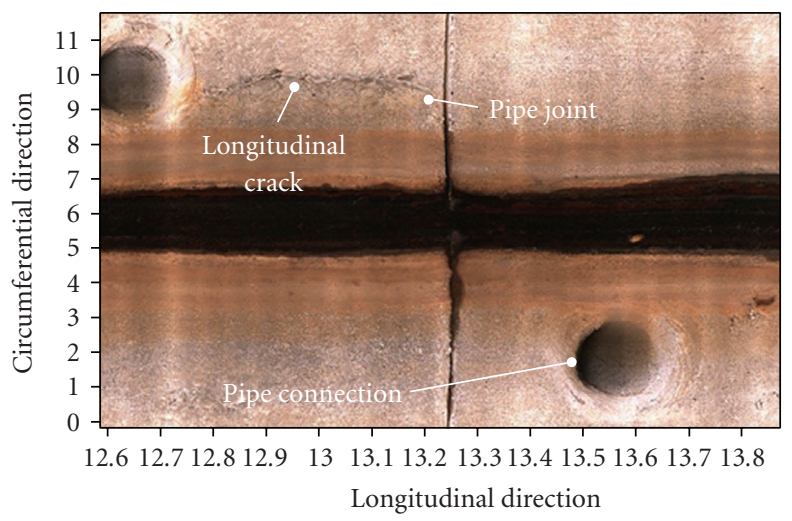

FIGURE 3: A section of an unwrapped pipe image.

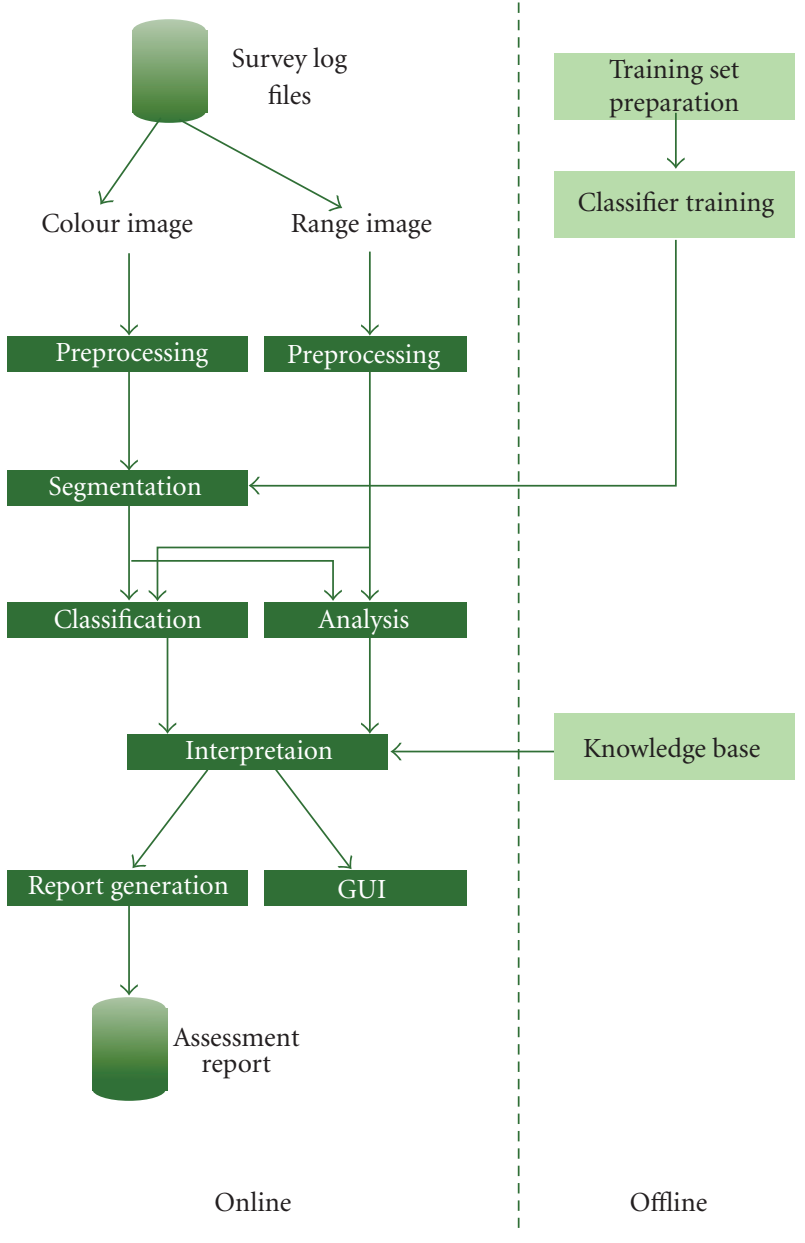

FIGURE 4: Schematic diagram of proposed system. colour and intensity, there may be no way of determining whether a defect or feature is an intrusion or an extrusion. This is because a patch of pipe surface looks essentially the same if it is translated in space. This means that it may be difficult to distinguish between categories such as "corrosion" and "deposit" for defects. On the other hand it maybe that corrosions tend to have a particular colour or texture signature while deposits have another in which case the colour image data would be sufficient to distinguish between them.

With range images it can be determined whether a defect or feature is an intrusion or an extrusion by simply comparing the range values on the defect with the nearby range values off the defect. For this reason we may also assume, when discussing properties related to range values of defects, that the interpretation system has an available unwrapped pipe range image. This range image may be provided by the inspection system or else possibly constructed by processing the colour images obtained by the inspection system using photogrammetry. If photogrammetry is used then the cost of an inspection device would only be the cost of a colour image acquisition system such as in a commercially available system. If the range image is to be obtained from the inspection device then the range image acquisition hardware can be easily integrated, at low cost, into existing camerabased inspection systems [14].

The proposed system is similar to that used in the PIRAT interpretation system. It consists of preprocessing, segmentation, classification, image analysis and high level system modules. Preprocessing carries out such operations as smoothing and filtering to put the pipe image in a suitable form for the subsequent processing modules. The segmentation module partitions the input image into meaningful subsets. In the case of a two-class segmentation each subset is either a "region of interest" (ROI) or "good pipe." Segmentation can be effected by pixel labelling combined with connected component labelling. The image classification module classifies each ROI output by segmentation as being in one of a number of classes such as "hole," "corrosion," "pipe connection," "deposit" and "tree root."

A basic difference between the proposed system and the PIRAT system is that the segmentation operates using colour digital images rather than range images. This opens up the possibility of more effective segmentation using colour information. A schematic diagram for the proposed system is given in Figure 4.

As a result of using colour image data is that it is difficult to generate suitable simulated data. This means 
that the pixel labelling classifier for segmentation and the image classifier have to be trained from real data. If neural networks or related classifiers are used then these classifiers require hundreds or thousands of training cases for training. For the case of the pixel labeller this may not be so bad because just one image contains many thousands of pixels.

However, for training the image classifier many hundreds of images would be required. This is unlikely to be feasible. Therefore the proposed system will not use image classification by neural network or similar classifier. Rather image classification can be carried out as part of image analysis or by using multiclass segmentation.

A decision tree classifier can be implemented. The fundamental decision that has to be made about an ROI is whether it is an intrusion or an extrusion. This can be made by comparing the average range value of on-defect pixels with the average range value of off-defect pixels which are in a neighbourhood of the boundary of the defect.

The difference of these values in the case when the defect is determined to be an extrusion can be used to assign the defect to being either a hole or corrosion. Alternatively, by using appropriate fuzzy membership functions, the defect can be assigned fuzzy membership function values in the classes "hole" and "corrosion."

Some holes can be identified as pipe connections by means of a pipe connection detector which examines the dimensions and location of the defect and may compute the ellipse of best fit for the boundary of the defect in order to give a compound fuzzy membership value.

Intrusive defects can be given a deterministic or fuzzy classification as either tree roots or deposits by use of a tree root detector which computes the maximum deviation between the range values of off-defect pixels and on-defect pixels in windows centred on boundary points of the defect.

Thus it is possible to construct a deterministic or fuzzy image classification module for the problem without using neural network classifiers.

The remainder of this paper describes some techniques for the recognition of defects and features in pipe images. The first step in each case is to carry out segmentation of the pipe image (see Section 5), which results in a binary image. The connected components of this binary image are candidates for defect regions or pipe features. The large connected component extending over the full length of the pipe is referred to as the principal segmented region and contains the pipe flow lines, the pipe joints, and adjoining defects. Other connected components are candidates for corrosion defects and pipe connections. An approach is therefore needed to distinguishing between corrosion regions and pipe connections. In this research, corrosion defects and pipe connections are distinguished using a simple fuzzy approach. The principal segmented region is then decomposed into its component flow lines region, pipe joint regions, and adjoining defect regions using the techniques of mathematical morphology, as described in Section 7. Generalisations of the morphological operations of erosion and dilation are described for this purpose.

\section{The Segmentation SubSystem}

There are a number of approaches to colour image segmentation including thresholding, feature-based clustering, region-based approaches, edge detection approaches, fuzzy approaches, and neural network approaches [15].

The simplest approach is to threshold the brightness and then to carry out connected component labelling. This has been carried out in the system of Moselhi and Shehab-Eldeen [16]. The resulting segmentation is dependent upon the threshold value chosen. A standard method for choosing a threshold is applicable when the image brightness histogram is bimodal at some level of resolution in the space of pixel values in which case the threshold is chosen at the location of the valley between the two peaks. However, experiments have shown that the brightness histograms for the pipe images are not always bimodal.

A threshold can be chosen by trial and error for a number of sample images and then tested more widely over a wider class of images. 23 trial images were taken of $393 \mathrm{~m}$ of concrete pipe. It was found that, while generally regions of corrosion were darker than regions of good pipe, some regions of good pipe were darker than some regions of corrosion. Therefore a single threshold cannot be used to distinguish between regions of corrosion and regions of good pipe. An approach to getting around this problem, and also the problem of images coming from different pipe types, is to preprocess regions of pipe images by applying image equalisation. An initial segmentation based on thresholding at the left-most valley in the image brightness histogram was carried out. This identified the darker regions of the pipe image resulting from the flow lines, pipe joints and pipe connections. Equalisation was carried out on the complement of the union of these regions in blocks. The blocks were chosen to be about $10 \%$ of the width of each pipe image, large enough to not be overly dominated by any particular defect or feature. It was found that the equalisation enhanced the random variations in the images and obscured the defects. Therefore, a simpler, linear transformation was used rather than the nonlinear equalisation transformation. The images in blocks were rescaled according to the average brightness of the image in the block outside the initially segmented dark regions. At each pixel in the block outside the initially segmented dark regions and for each colour component the value is changed according to

value $=$ old $_{\text {value }} \times($ reference_brightness / average_brightness $)$,

where reference_brightness can be chosen to be the average brightness of a "typical" image in the set of available pipe images.

The blockwise rescaled images were globally thresholded. In this process a change to the system parameter reference_brightness is equivalent to a corresponding change in the threshold. Therefore the parameter reference_brightness does not act as an independent system parameter and the value chosen for it is not significant as long as the threshold value can be chosen subsequently. A resulting segmentation 
is obtained by taking the connected components of the label image resulting from the thresholding where, for any pixel,

$$
\text { label }=1 \text { if brightness }<\text { threshold, } 0 \text { otherwise, }
$$

in which the brightness is the maximum of the red, green, and blue values for the pixel.

Each threshold value is associated with a label image which defines a subset of the image lattice consisting of those pixels for which the label is 1 . The map from threshold values to subsets of the image lattice is a homomorphism into the lattice of subsets ordered by inclusion. In other words, given two thresholds threshold_1 and threshold_2 and corresponding subsets subset_1 and subset_2 we have

$$
\text { (threshold_1 }<\text { threshold_2 }) \Longrightarrow(\text { subset_1 } \subset \text { subset_2). }
$$

Experiments show that when the threshold is large the associated label image is large, resulting in oversegmentation while if the threshold is small the associated label image is small resulting in under-segmentation. Oversegmentation manifests as false positives in the found defect regions, that is regions which are flagged as being defect regions which do not appear to be defect regions. Undersegmentation manifests as false negatives, that is regions which appear to be defect regions but are not so flagged. It is found that for some images both false positives and false negatives are present in the segmentation. Therefore there is no threshold value which results in a completely correct segmentation for all images.

The main segmentation approach that we consider in this paper is described in [17]. It is a supervised method involving classification of feature vectors derived from pixels or neighbourhoods of pixels [18].

A number of authors have used a feature vector consisting simply of the RGB (red, green, and blue) values of a pixel as inputs to a classifier [19]. We have also used as a feature set the $\mathrm{H}, \mathrm{S}$, and B components in the HSB (hue, saturation, and brightness) colour space [20]. In order to obtain a segmentation system which is independent of lighting conditions we have also tried a feature set consisting of the $\mathrm{H}$ and $\mathrm{S}$ components of the HSB colour space.

In addition, a slightly more sophisticated feature set has been implemented in which the $\max , \min$, and average are computed for RGB values in a window (of size say $7 \times 7$ ) about each pixel together with the max, min, and average of an intensity feature I given by

$$
I=\sqrt{\left(R_{2}+G_{2}+B_{2}\right)} .
$$

Also we have used a more elaborate feature set constructed from Gabor texture filter banks [21, 22]. For the classifier we used an SVM. SVMs have certain properties that make them superior to neural networks such as requiring smaller training sets and having better generalisation ability.

For generation of a training set for the SVM a set of feature vectors for individual pixels together with their classifications must be extracted from the training data images. The feature vectors can be extracted automatically but the classification labels must be determined manually (if they could be determined automatically then we would have already solved the problem of segmentation).

This manual pixel classification process can be effected by using a GUI program which displays a training image and enables the user to select certain regions or sets of pixels and specify that they are of a particular classification.

A simpler approach to generating training sets for the pixel classifier is to select rectangular regions of constant classification using standard tools. This simpler approach was used, the rectangular regions shown in Figure 5 were selected and extracted from the unwrapped pipe image of a concrete sewer pipe (part of which is shown in Figure 3). The regions were extracted from regions of corrosion and regions of good pipe. The files good_1.bmp and good_2.bmp were used for testing the classifier while the remaining corrosion and good pipe files were used for both training and testing.

Training and testing using this data was carried out for each of the feature sets. The results of testing the pixel classifier on the testing sets that had been set aside are shown in Table 1. The table shows that when tested on the testing data that had been set aside during training and on the data contained in good_1.bmp and good_2.bmp the classifier performed well when using all feature sets except the HS feature set.

In applying the SVM pixel classifiers generally to images other than those used in training the blockwise rescaling described above was applied. In order to carry out wider testing, the SVM pixel classifier algorithms for the RGB, Gabor, local window, HSB and HS feature sets were applied to the images taken from $393 \mathrm{~m}$ of concrete sewer pipe. The result for the HSB case applied to the image of Figure 3 is shown in Figure 6. The best segmentation results were obtained for the HSB feature set [17].

The RGB, Gabor, and HSB pixel labelling images contain single pixel and other very small defects. These are perhaps not a problem because such defects can be filtered out at a higher level by the pipe inspection system during image classification and analysis.

\section{Detection of Corrosion Defects and Pipe Connections}

The segmentation operation results in a number of regions for each pipe image. The largest such region which extends over the full length of the pipe is made up from the flow lines at the bottom of the pipe together with any pipe joints radiating out from the flow lines. Also any defective regions which are adjoined to these regions form part of this principal segmented region. Segmented regions other than the principal segmented region form candidates for corrosion regions, other defect regions or pipe connections. In the work of this paper we are concentrating on the defect type of corrosion.

Pipe connections may be distinguished from corrosion regions by using a simple fuzzy approach. A defect region is likely to be a pipe connection if it is located approximately laterally in the pipe and its size is within a range of suitable 


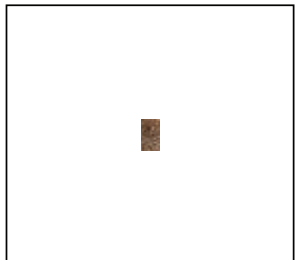

Corrosion_1.bmp

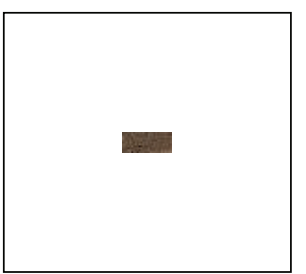

Corrosion_7.bmp

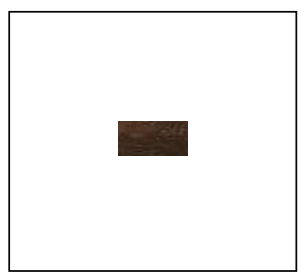

Corrosion_13.bmp

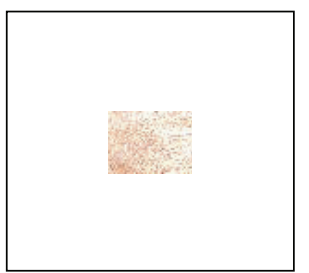

Good_6.bmp

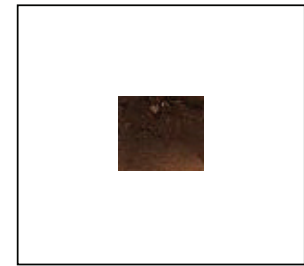

Corrosion_2.bmp

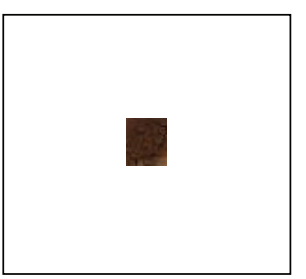

Corrosion_8.bmp

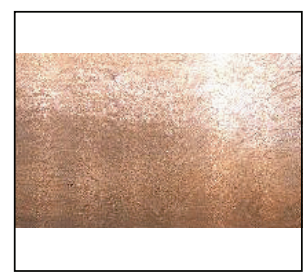

Good_1.bmp

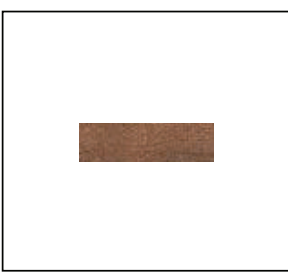

Good_7.bmp

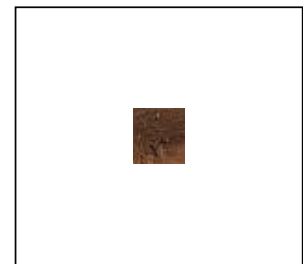

Corrosion_3.bmp

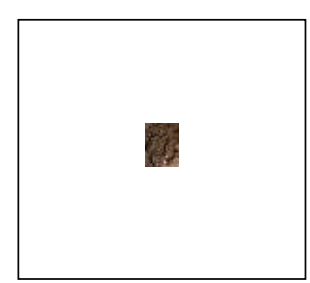

Corrosion_9.bmp

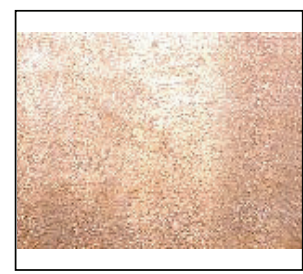

Good_2.bmp

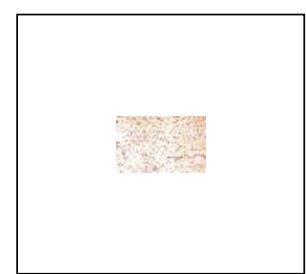

Good_8.bmp

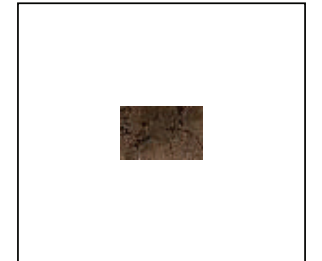

Corrosion_4.bmp

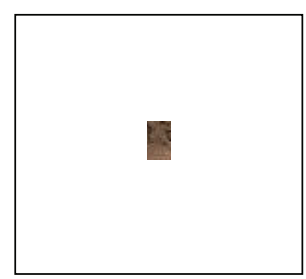

Corrosion_19.bmp

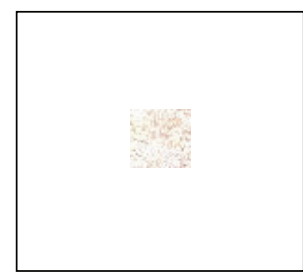

Good_3.bmp

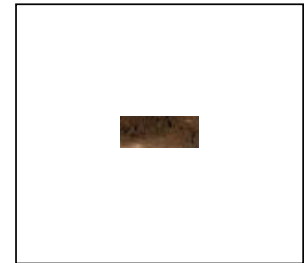

Corrosion_5.bmp

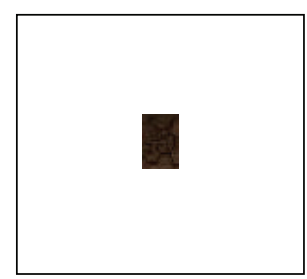

Corrosion_11.bmp

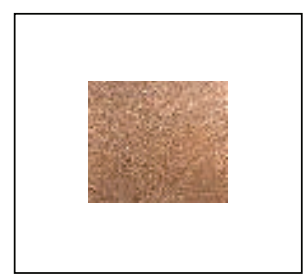

Good_4.bmp

FIGURE 5: Rectangular regions of corrosion and good pipe.

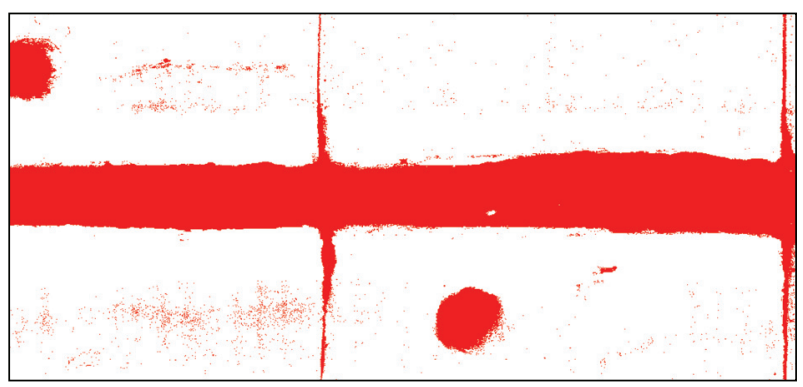

Figure 6: HSB-SVM Pixel labeling.

sizes. One may also consider requiring that its shape as measured by, for example, a goodness of fit parameter for an ellipse of best fit is in a suitable range. However, as noted by Müller and Fischer [23] segmented regions arising from pipe connections can come in a number of shapes other than oval.
One may define fuzzy membership functions associated with the conditions that the defect is laterally located and of a suitable size. Such membership functions are shown in Figure 7 in which $\theta$ is the angle of the centroid of the defect in the pipe measured from the bottom of the pipe and $\mathrm{A}$ is the area of the defect in pixels. The centroid of a defect is computed automatically by taking the vector average of the position vector of pixels in the defect. The angle of the centroid is computed automatically by transforming the $y$ coordinate of the centroid to lie in the interval $(-180,180]$. The area of a defect is computed automatically by counting the number of pixels in the defect. In line with the usual rule for combining membership functions under conjunction an overall membership function value for whether or not the defect is a pipe connection may be obtained by taking the minimum of these two membership function values. A membership function value for whether the defect is corrosion may be obtained by taking the complement of the pipe connection membership value. A 


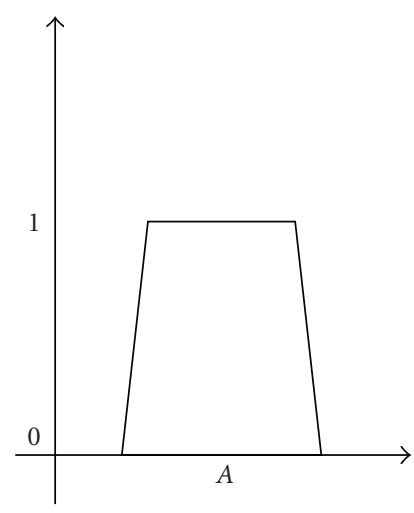

(a)

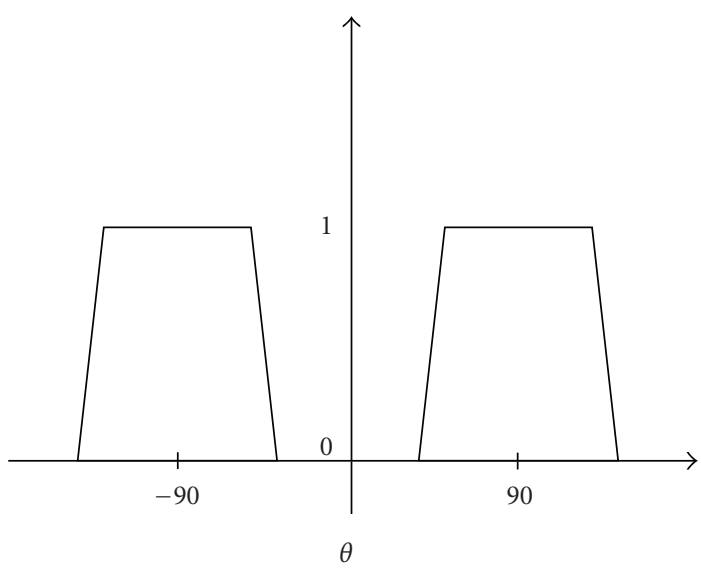

(b)

FIGURE 7: Form of membership functions for pipe connection size and location.

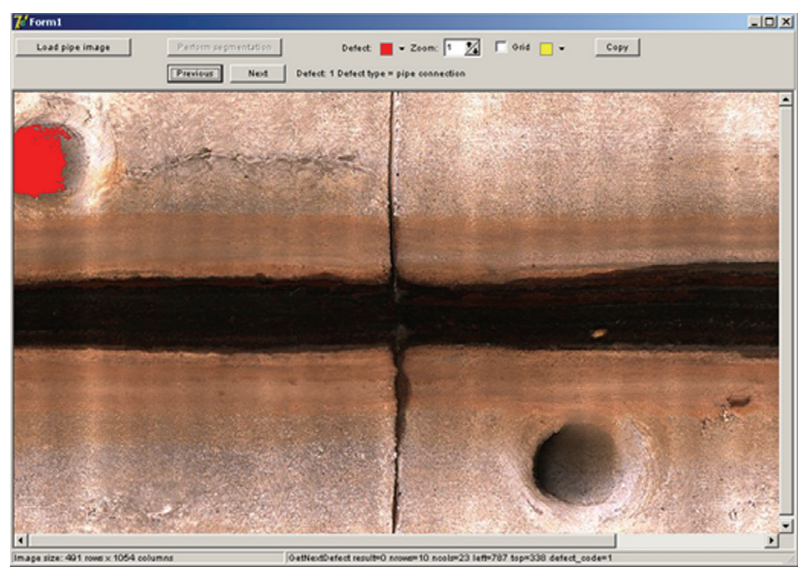

FIGURE 8: GUI display of pipe connection.

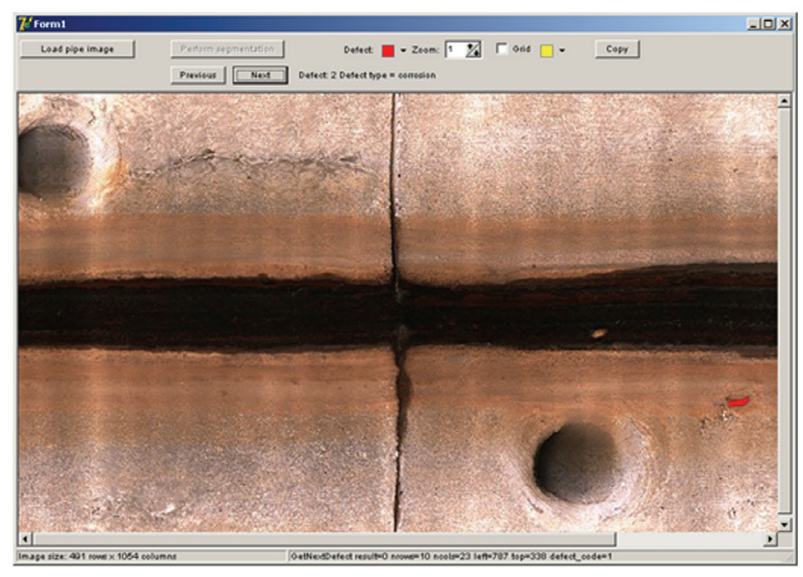

FIGURE 9: GUI display of corrosion defect.

defect may be declared to be a pipe connection if its pipe connection membership function value exceeds its corrosion membership function value, and corrosion otherwise. Pipe connection detection by this simple fuzzy approach has been applied to the trial images under consideration with the result of completely accurate detection except in one case where the pipe connection was on the boundary of the image. Cases such as these can be avoided by processing the full unwrapped pipe image or else by using overlapping windows.

Defect regions together with their classification as either corrosion or pipe connection can be displayed by means of a GUI as shown, for example, in Figures 8 and 9 where the results of SVM segmentation using HSB feature vectors are displayed.

The effectiveness of the system at finding defects or features depends upon the quality of the segmentation. For the SVM segmentation the result depends upon the parameter reference_brightness which is significant because the SVM training is done without rescaling the pixel values. In this case the value of reference_brightness is determined by trial and error. When reference_brightness is high the system under-segments resulting in more false negatives. When reference_brightness is low the system over-segments resulting in more false positives. Some values of reference_brightness are associated with both false positives and false negatives. Thus, as in the case of the thresholding method, perfect segmentation cannot be achieved using the SVM method.

However, the SVM method has the potential to be a more powerful segmentation method because it takes into account the colour properties of the images rather than just the greyscale brightness value and so is utilising more information present in the image. For the remainder of this paper the segmentation method used is SVM pixel classification using the HSB feature vectors.

\section{Detection of Flow Lines, Pipe Joints, and Adjoining Defects}

It is desirable to have a method of decomposing the principal segmented region into its separate components, that is, flow lines, pipe joints, and adjoining defects. This can be achieved using the methods of mathematical morphology. 
TABLE 2: Classifier test results.

\begin{tabular}{lccccc}
\hline Feature set & No. of training cases & No of testing cases & \% accuracy of testing & \% accuracy on good_1.bmp & \% accuracy on good_2.bmp \\
\hline RGB & 3000 & 17867 & 96.88 & 99.15 & 99.93 \\
HSB & 1000 & 19867 & 97.30 & 99.39 & 99.98 \\
Gabor & 5000 & 15867 & 97.11 & 99.25 & 99.92 \\
Window & 3999 & 10100 & 99.83 & 99.98 & 100.00 \\
HS & 155000 & 19867 & 67.81 & 64.11 & 92.61 \\
\hline
\end{tabular}

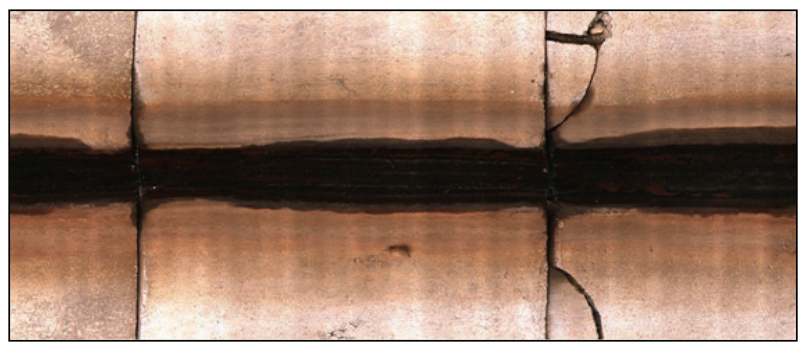

FIGURE 10: Typical example of flow lines, pipe joints, and adjoining defects.

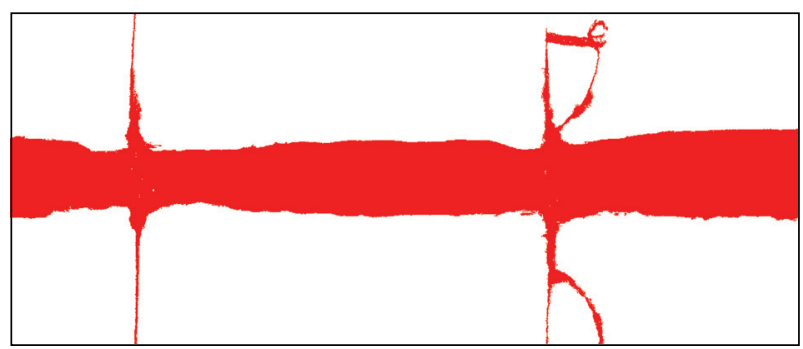

Figure 11: Principal binary image.

Grey-scale morphology has been used by Sinha and Fieguth [24] to segment pipe joints, and pipe connections. However, in our work, a binary segmented image is already present as a result of prior segmentation using SVM or other method. Therefore the more reliable binary image morphology can be used. Also, we define generalisations of the morphology operations which are more suitable to the images being analysed.

Consider the problem of flow line detection. The image of Figure 10 is a typical example of a section of pipe containing flow lines, pipe joints and adjoining defects. The associated principal binary image obtained after SVM segmentation and connected component labelling is shown in Figure 11. Now we recall that if a binary image is associated with a set $A \subset X$ where $X=\{0, \ldots, m-1\} \times\{0, \ldots, n-1\}$ is the image lattice then the erosion $A \ominus E$ of $A$ with respect to a structuring element $E \subset \mathbf{Z}^{2}$ is given by

$$
A \ominus E=\left\{x \in X: E_{x} \cap X \subset A\right\},
$$

and the dilation $A \oplus E$ of $A$ with respect to $E$ is given by

$$
A \oplus E=\left\{x \in X: \widehat{E}_{x} \cap A \neq \varnothing\right\},
$$

where

$$
E_{x}=\{x+e: e \in E\} \subset \mathbf{Z}^{2}, \quad \widehat{E}=\{-e: e \in E\} .
$$

Let $A$ be the principal segmented region shown in Figure 11. It is natural to try to define the core region of the flow lines to be given by $A \ominus E$ where $\mathrm{E}$ is the horizontal structuring element given by

$E=\{(0, j): j=-$ element_length_1 $1, \ldots$, element_length_1 $\}$.

However, the result of this erosion with element_length_1 $=100$ is shown in Figure 12. The erosion does not provide a good representation of the flow line region. The reason for this behaviour is that some points in the convex hull of the flow line region are missing due to random noise variation. This results in the lines of missing values in the eroded image. This behaviour can be avoided by defining a generalisation of the erosion operation which we call $\alpha$-erosion, fractional erosion, or partial erosion. Let $\alpha \in[0,1]$. The $\alpha$-erosion [25] $A \ominus_{\alpha} E$ of A by a structuring element $E$ is defined by

$$
A \ominus_{\alpha} E=\left\{x \in X:\left|E_{x} \cap A\right| \geq \alpha\left|E_{x} \cap X\right|\right\},
$$

where, for a set $S,|S|$ is the number of elements of $S$. Thus $A \ominus_{\alpha} E$ consists of all those points $x$ in $X$ such that the fraction of points in the $x$-translate of $E$ which are in $A$ is greater than or equal to $\alpha$.

Theorem 1. For $\alpha=1$ the $\alpha$-erosion $\ominus_{\alpha}$ coincides with the usual erosion $\ominus$. A proof of this theorem is given in [25]. It is also straightforward to show that

$$
\alpha<\beta \Longrightarrow A \ominus_{\alpha} E \supset A \ominus_{\beta} E,
$$

and that

$$
A \ominus_{0} E=X .
$$

One may also define, for $A \subset X, E \subset \mathbf{Z}^{2}$, and $\alpha \in[0,1]$, the $\alpha$-dilation $A \oplus_{\alpha} E$ of $A$ by $E$ by

$$
A \oplus_{\alpha} E=\left\{x \in X:\left|\widehat{E}_{x} \cap A\right|>\alpha\left|E_{x} \cap X\right|\right\} .
$$

Clearly, for $\alpha=0$ the $\alpha$-dilation $\oplus_{\alpha}$ coincides with the usual dilation $\oplus$, for $\alpha=1 A \oplus_{\alpha} E=\varnothing$ and $\alpha<\beta \Longrightarrow A \oplus_{\alpha} E \supset$ $A \oplus_{\beta}$ E. Also, one has the following duality theorem connecting $\oplus_{\alpha}$ and $\ominus_{\alpha}$. 


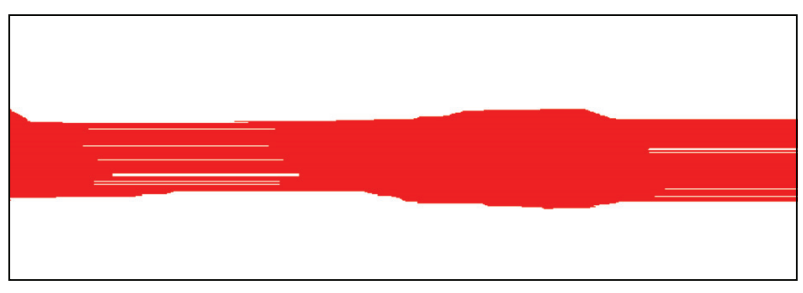

FIGURE 12: Erosion of principal binary image.

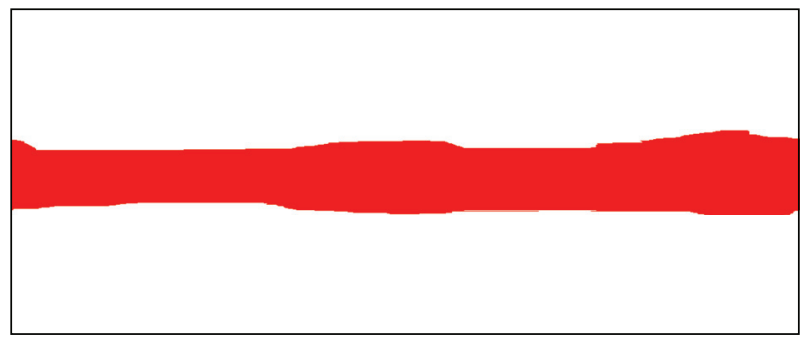

FIGURE 13: Core flow line region $=\alpha$-erosion of principal binary image with $\alpha=0.9$.

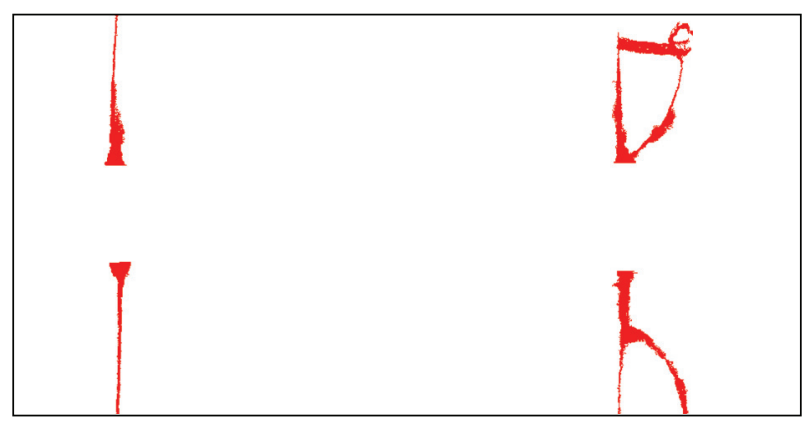

FIGURE 14: Principal binary image core flow line region.

Theorem 2. There is

$$
\sim\left(A \ominus_{\alpha} E\right)=\sim A \oplus_{1-\alpha} \hat{E}
$$

A proof of this theorem is given in [25].

The result of carrying out the $\alpha$-erosion of the image in Figure 11 by the horizontal structuring element defined above with element_length_1 $=100$ and $\alpha=0.9$ is shown in Figure 13. This gives a good representation of the core of the flow line region. The flow line region may be taken to be estimated by the dilation of the core flow line region by the structuring element

$E=\left\{(i, 0): i=-\right.$ element_length $\_2, \ldots$, element_length $\left.\_2\right\}$.

The binary image obtained by taking $P \sim F$ where $P$ is the principal binary image and $F$ is the (estimated) flow line region is shown in Figure 14. This represents the pipe joints and adjoining defects. Candidate pipe joints may be

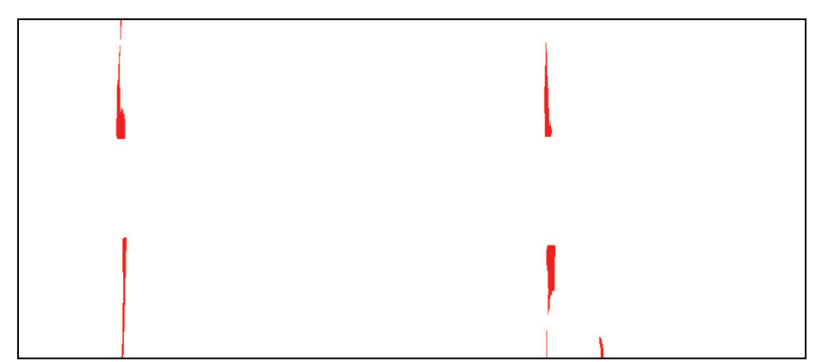

FIgURe 15: Candidate pipe joints.

detected by taking the $\alpha$-erosion of this image with respect to the structuring element

$E=\{(i, 0): i=-$ element_length_3, . , element_length_3 $\}$,

with the result shown in Figure 15.

The candidate pipe joint components are the connected components of this image. In the case of the given example, there are 6 such components. Pipe joints may be determined by analysis of the candidate pipe joint set [25].

The method of pipe joint detection described above has been applied to 23 images of $393 \mathrm{~m}$ of concrete pipe under consideration with the result of $100 \%$ classification accuracy.

\section{Conclusions}

Automation of pipe inspection has the potential to improve the efficiency of the CCTV inspection process through the use of fully or partially automated systems. A semiautomatic pipe inspection system can act as a screening system filtering out images obtained by the image acquisition system which definitely (or to a very high degree of probability) contain no significant defects. A useful function of an automated pipe inspection system is to be able to detect and recognise pipe features such as pipe connections, flow lines, and pipe joints as well as pipe defects.

In this paper an approach to signal interpretation for automatic pipe inspection based on segmentation of colour images by SVM has been presented. The segmentation approach has been tested using RGB, Gabor, local window, HSB, and HS feature sets. It has been found that the method works best in the case of the HSB feature set. The segmentation algorithm can be used as part of a pipe inspection system utilising also range image information for the purpose of defect classification. Such range image information can be obtained either directly from the inspection device or else possibly by analysing colour images obtained from the inspection device by using multiple viewpoint geometry.

A simple approach to detecting pipe connections using fuzzy membership functions relating to defect size and location has been described.

Analysis of the principal segmented region by mathematical morphology enables the flow line region, pipe joints, and adjoining defects to be detected. While manifesting some false positives or false negatives in corrosion detection, the 
system had essentially $100 \%$ accuracy in the detection of flow line region, pipe joints and pipe connections on the data on which it was tested.

\section{Acknowledgments}

The authors thank Mike Rahilly for implementing the graphical user interface and for very helpful discussions, Donavan Marney for very helpful discussions, Tristan Day for supplying colour images of sewers, and Scott Gould for help with the selection of pipe defect images.

\section{References}

[1] D. Marlow, S. Heart, S. Burn, et al., Condition Assessment Strategies and Protocols for Water and Wastewater Utility Assets, WERF, Alexandria, Va, USA, 2007, Project Ref 03-CTS-20CO.

[2] S. Burn, D. Marlow, M. Moglia, and P. Buckland, "Asset management for urban infrastructure," Water Asset Management International, vol. 3, no. 2, pp. 12-18, 2007.

[3] D. Marlow and S. Burn, "Effective use of condition assessment within asset management," Journal of the American Water Works Association, vol. 100, no. 1, 2008.

[4] R. P. Hoskins, A. T. Brint, and G. Strbac, "A structured approach to asset management within the electricity industry," Utilities Policy, vol. 7, no. 4, pp. 221-232, 1999.

[5] J. P. Davies, B. A. Clarke, J. T. Whiter, and R. J. Cunningham, "Factors influencing the structural deterioration and collapse of rigid sewer pipes," Urban Water, vol. 3, no. 1-2, pp. 73-89, 2001.

[6] S. E. McDonald and J. Q. Zhao, "Condition assessment and rehabilitation of large sewers," in Proceedings of the International Conference on Underground Infrastructure Research, pp. 361-369, University of Waterloo, Waterloo, Canada, June 2001.

[7] Sewer Rehabilitation Manual (SRM), Version 2, WRc, 4th edition, 200.

[8] W. Guo, L. Soibelman, and J. H. Garrett Jr., "Automated defect detection for sewer pipeline inspection and condition assessment," Automation in Construction, vol. 18, no. 5, pp. 587-596, 2009.

[9] H. Kuntze, H. Haffner, M. Selig, D. Schmidt, K. Janotta, and M. Loh, "Development of a flexible utilisable robot for intelligent sensor-based sewer inspection," in Proceedings of the 4th International Conference on Pipeline Construction, pp. 367374, Hamburg, Germany, 1994.

[10] R. Kirkham, P. D. Kearney, K. J. Rogers, and J. Mashford, "PIRAT—a system for quantitative sewer pipe assessment," International Journal of Robotics Research, vol. 19, no. 11, pp. 1033-1053, 2000.

[11] J. S. Mashford, "A neural network image classification system for automatic inspection," in Proceedings of the IEEE International Conference on Neural Networks, vol. 2, pp. 713-717, Perth, Australia, 1995.

[12] O. Moselhi and T. Shehab-Eldeen, "Classification of defects in sewer pipes using neural networks," Journal of Infrastructure Systems, vol. 6, no. 3, pp. 97-104, 2000.

[13] S. K. Sinha and F. Karray, "Classification of underground pipe scanned images using feature extraction and neuro-fuzzy algorithm," IEEE Transactions on Neural Networks, vol. 13, no. 2, pp. 393-401, 2002.
[14] O. Duran, K. Althoefer, and L. D. Seneviratne, "Automated pipe defect detection and categorization using caniera/laserbased profiler and artificial neural network," IEEE Transactions on Automation Science and Engineering, vol. 4, no. 1, pp. 118126, 2007.

[15] H. D. Cheng, X. H. Jiang, Y. Sun, and J. Wang, "Color image segmentation: advances and prospects," Pattern Recognition, vol. 34, no. 12, pp. 2259-2281, 2001.

[16] O. Moselhi and T. Shehab-Eldeen, "Automated detection of surface defects in water and sewer pipes," Automation in Construction, vol. 8, no. 5, pp. 581-588, 1999.

[17] J. Mashford, P. Davis, and M. Rahilly, "Pixel-based colour image segmentation using support vector machine for automatic pipe inspection," in Proceedings of the 20th Australian Joint Conference on Artificial Intelligence (AI '07), vol. 4830 of Lecture Notes in Computer Science, pp. 739-743, Springer, Gold Coast, Australia, 2007.

[18] W. E. Blanz and S. L. Gish, "A real-time image segmentation system using a connectionist classifier architecture," International Journal of Pattern Recognition and Artificial Intelligence, vol. 5, no. 4, pp. 603-617, 1991.

[19] H. Gómez-Moreno, P. Gil-Jiménez, S. Lafuente-Arroyo, R. Vicen-Bueno, and R. Sánchez-Montero, "Color images segmentation using the support vector machines," Recent Advances in Intelligent Systems and Signal Processing, pp. 151155, 2003.

[20] R. Gonzalez and R. Woods, Digital Image Processing, PrenticeHall, Upper Saddle River, NJ, USA, 2nd edition, 2002.

[21] S. E. Grigorescu, N. Petkov, and P. Kruizinga, "Comparison of texture features based on Gabor filters," IEEE Transactions on Image Processing, vol. 11, no. 10, pp. 1160-1167, 2002.

[22] T. Randen and J. H. Husøy, "Filtering for texture classification: a comparative study," IEEE Transactions on Pattern Analysis and Machine Intelligence, vol. 21, no. 4, pp. 291-310, 1999.

[23] K. Müller and B. Fischer, "Objective condition assessment of sewer systems," in Proceedings of the 2nd Leading Edge Conference on Strategic Assessment Management (LESAM '07 ), Lisbon, Portugal, 2007.

[24] S. K. Sinha and P. W. Fieguth, "Segmentation of buried concrete pipe images," Automation in Construction, vol. 15, no. 1, pp. 47-57, 2006.

[25] J. Mashford, M. Rahilly, and P. Davis, "An approach using mathematical morphology and support vector machines to detect features in pipe images," in Proceedings of the IEEE Digital Image Computing: Techniques and Applications (DICTA '08), pp. 84-89, Canberra, Australia, 2008. 

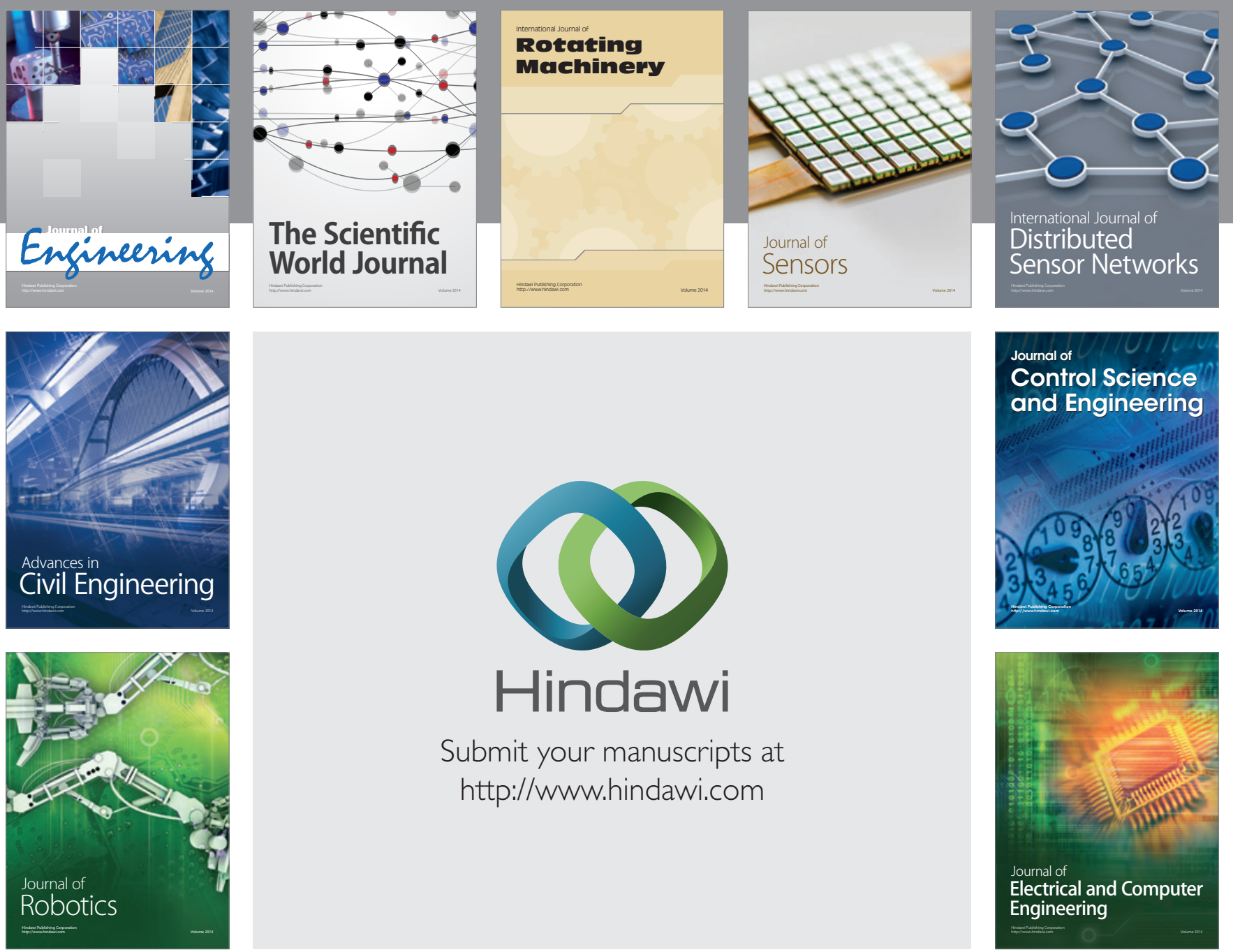

Submit your manuscripts at

http://www.hindawi.com
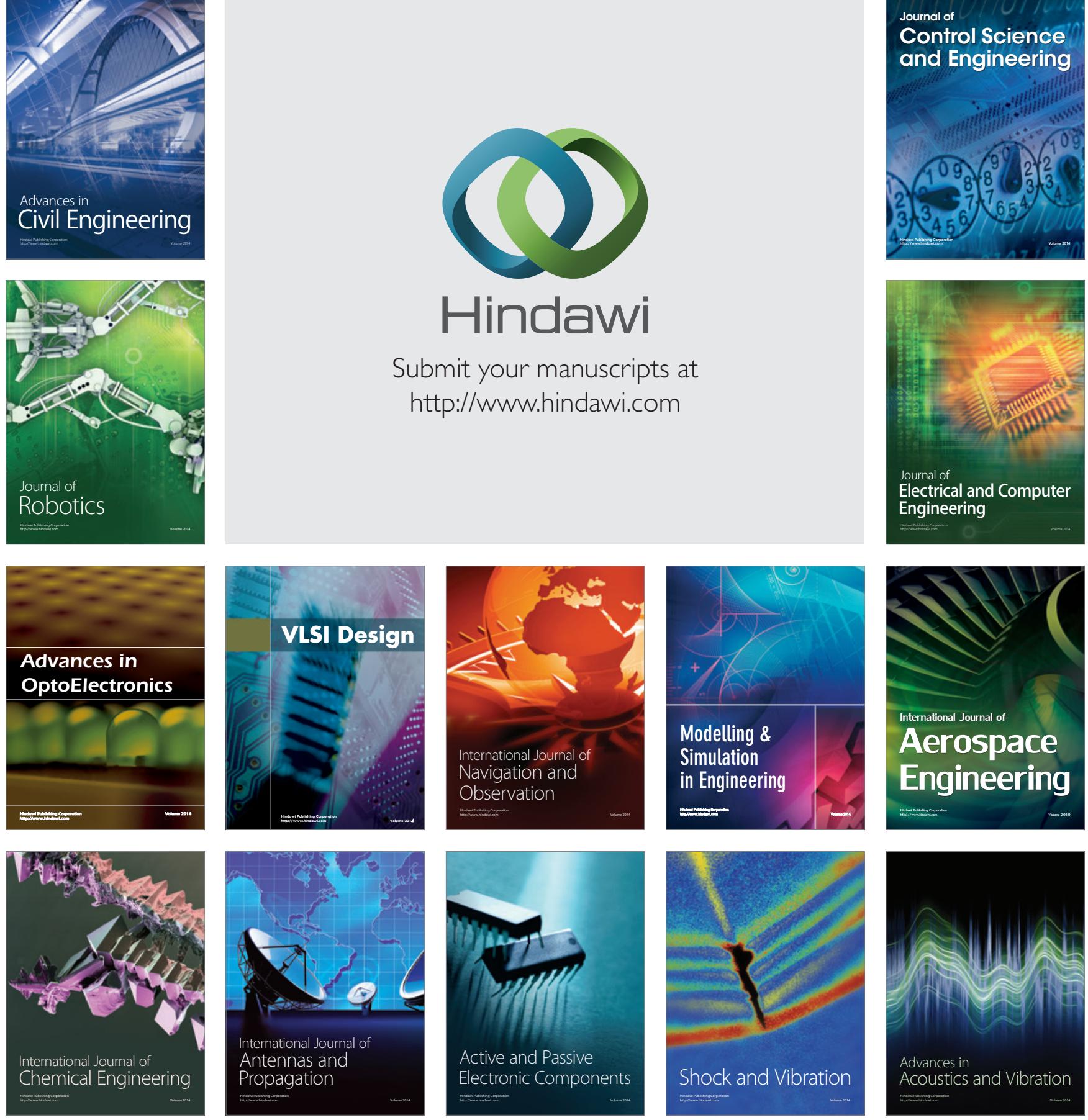